\title{
Análisis inyuntivo de la interacción: Un sistema de categorías (S.A.I.I.)
}

\author{
Yolanda Gómez FonTANIL \\ Universidad de Oviedo \\ Esteban Coto Ezama \\ Centro de Investigaciones Comunicacionales. Oviedo
}

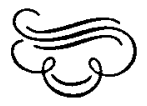

\section{Resumen}

En este artículo se expone un nuevo sistema de análisis de la interacción, basado en los análogos de la petición y oferta de objetos y de la aprehensión, conservación y uso de instrumentos, diseñado para dar cuenta de procesos de control interpersonal en las interacciones cara a cara. Se dan muestras de la aplicación de sus categorias y se ofrecen pruebas de su fiabilidad.

Palabras clave: Análisis de la interacción. Categorización.

\section{Abstract}

In this article we expose a new system of analysis of interaction, based on the analogous of demand and offer of objects and the aprehension, conservation and use of instruments, designed to give account of interpersonal ocntrol or processes in the face to face interactions. We give samples of the application of its categories and we offer reliability tests.

Key words: Interaction analysis. Categorization.

Dirección de los autores: Universidad de Oviedo. Departamento de Psicología. 


\section{PRESENTACION}

Desde finales de los años cincuenta el análisis de la interacción se ha ido convirtiendo poco a poco en un instrumento de uso corriente en el ámbito de la Salud Mental (Haley, 1977; Sluzki y Beavin, 1965; Riskin y Faunce, 1970; Gottman, 1979; Rogers, Courtright y Millar, 1979; Viaro y Leonardi, 1982; Miklowitz, Goldstein, Doane, Nuechterlein, Strachan Snyder y MagañaAmato, 1989). En el de la Psicología Evolutiva el paso de los denominados modelos de competencia a los modelos procesales, ha hecho de la interacción efectiva entre adultos y niños y de la interacción entre pares, áreas de trabajo sumamente productivas en las dos últimas décadas (Ervin Tripp, 1977; Kaye y Fogel, 1980; Bruner, 1986). Idéntico auge, o aun mayor, experimenta el estudio de la interacción en Psicología Escolar, tras la irrupción de la Lingüística, la Etnometodología, la Microsociología y la Sociolingüística, en el aula y con el resurgir de las ideas vygotskyanas en Psicología Evolutiva (Sinclair y Coulthard, 1975; Stubs, 1987; Edwards y Mercer, 1988). Y en Psicología Social, Harré y sus colaboradores, con su hincapié en el análisis de los episodios sociales, han hecho de la conversación una realidad básica a estudiar Harré y Secord (1972), Harré (1982), Harré, Clarke y De Carlo (1989).

En este artículo exponemos un nuevo sistema para el estudio de procesos de interacción comunicativa, diseñado sobre dos modelos analíticos articulados en un trabajo anterior (Gómez Fontanil y Coto Ezama, 1990): la petición y oferta de objetos y la aprehension, conservación y uso de instrumentos.

Un sistema de análisis de la interacción puede ser considerado como un mero instrumento para contrastar hipótesis, así lo hacen Bakeman y Gottman (1989), por ejemplo, pero también puede ser visto como la reconstrucción de un fenómeno que el lenguaje cotidiano construye vaga e insuficientemente. En las interacciones cara a cara, y especialmente en las conversaciones, ocurren demasiadas cosas de las que no sabemos hablar, demasiadas cosas que se distinguen "por la impresión que uno tiene», como para que podamos conformarnos con la realidad que nos da el lenguaje común. El S.A.I.I. se ha construido como un instrumento para hacer distinciones inéditas y su aplicación produce una "realidad inédita». Sin embargo, el sistema refleja demasiado pálidamente los problemas que el análisis de la interacción plantea. Se ha construido sobre lo que creemos una «simplificación pertinente» y sólo la lectura del artículo antes citado (Gómez Fontanil y Coto Ezama, 1990) permitirá que se le haga justicia.

Nuestro sistema está fuertemente inspirado en la tradición del Grupo de Palo Alto, y en especial en el trabajo de Sluzki, Beavin, Tarnopolsky y Verón de 1966 titulado "Transacciones descalificadoras. Investigación sobre el "doble vínculo"». Aunque nos apoyemos en otras tradiciones, como la Pragmática Lingüistica de inspiración austiniana y el llamado Análisis del Discurso (Austin, 1982; Fabbri y Sbisà, 1980; Lozano, Peña-Marín y Abril, 1982; Sbisà, 1984; Stubs, 1987; Valdés Villanueva, 1983), el aire de familia no se le escapará a nadie que conozca ese precioso artículo. Como a sus autores nos ha interesado estudiar las interacciones cara a cara como secuencias de control interpersonal, como cadenas de «mandatos» (inyunciones), "obediencias» $\mathrm{y}$ "desobediencias».

Habiendo padecido con demasiada frecuencia la parquedad en ejemplos de otros autores, hemos querido ser generosos con las muestras de codificación, aun a costa de no tener espacio para dar una muestra de su utilidad. Hemos 
querido que el artículo pudiese servir como un verdadero «manual del codificador» y, aun siendo conscientes de que ello va en detrimento de la «comercialidad", para ellos hemos sacrificado la exposición de las aplicaciones ya realizadas. Debemos advertir, sin embargo, que emplear el sistema no es tarea fácil. Exige mucho entrenamiento y romper con hábitos comunicativos muy arraigados. Codificar eficientemente exige una disciplina cuidadosa que sólo se adquiere con entrenamiento y persistencia, pero confiamos en que ser tan prolijos les ahorrará a los interesados dudas y consultas.

Para emplear el sistema es imprescindible contar con un registro magnetoscópico y con una transcripción del diálogo. El registro en vídeo es absolutamente necesario para las categorías con componentes no verbales y para aumentar la fiabilidad en la categorización de las llamadas «conductas negativas»: sarcasmos, ironías... (Metz, 1965; Waxler y Mishler, 1966; Penman, 1980) y la transcripción es muy recomendable para facilitar la segmentación de las unidades y para evitar errores de interpretación. Los ejemplos que transcribimos provienen de un estudio sobre la eficacia de entrevistas terapéuticas (Gómez Fontanil, 1989). La Tabla I presenta las claves utilizadas en ellos.

TABLA I

Claves de transcripción

- Interrupción de palabra.

\# Sonido inarticulado

$=$ Alargamiento de la vocal o consonante anterior

$>$ Repetición de la sílaba anterior

» Repetición de la palabra anterior

(...) Fragmento sin descifrar

1 Pausa de menos de dos segundos

// Pausa de más de dos segundos

despis $\int \begin{aligned} & \text { te vamos }=\text { Interrupción de otro intelocutor con superposición } \\ & \text { y por otro lado }\end{aligned}$

\section{CATEGORIAS DEL S.A.I.I.}

\subsection{Segmentación de unidades}

En 1970, Simon y Boyer identificaban ocho criterios de segmentación de unidades diferentes en los estudios sobre conductas comunicativas, éstos eran: el cambio de categoría, el cambio de emisor, el cambio de tema, el cambio de audiencia, el cambio de lenguaje, la unidad pregunta-respuesta, unidades temporales y muestreo de tiempo. Desde 1970 hasta aquí se han seguido usando esos mismos criterios, aunque se ha dado mayor preponderancia a una unidad que, según quien la utilice (filósofos, semiólogos o psicólogos), 
recibe el nombre de «actos de habla» (speech act), «enunciado» (utterance) o «unidad de pensamiento» (Penman, 1980; Gottman, 1979; Mishler y Waxler, 1968).

Nuestra unidad mínima combina cinco de los criterios recogidos por Simon y Boyer. El término «movimiento» que elegimos para designarla proviene de la tradición goffmaniana. Para Goffman un movimiento es una unidad de análisis no esencialmente lingüística y puede consistir tanto en una secuencia discursiva como en una actividad física o en un fenómeno paralingüístico. Así la respuesta del atleta al mensaje: « $\mathrm{A}$ sus puestos!, ipreparados! ¡ya!» aún no siendo lingüística sigue siendo un «movimiento» al insertarse en un esquema interaccional. En este mismo sentido el silencio tras una pregunta puede interpretarse como un movimiento en sentido pleno.

Nuestra unidad mínima es el movimiento y los criterios por los que se segmenta y analiza tienen relación con las distintas posiciones que los interlocutores ocupan en el discurso, las posiciones que ocupan sus actividades y la propia composición de esta actividad. Respecto al canal verbal los interlocutores intercambian sus posiciones a lo largo de la interacción. Son en un momento destinadores, luego destinatarios y en otra ocasión audiencia. Sus emisiones adquieren también varias funciones: la de propuesta, la de respuesta y la de réplica. Al conjunto de componentes verbales y no-verbales que ocupan estas posiciones lo denominamos "movimiento", pero esta unidad no coincide totalmente con los mensajes emitidos por un interlocutor hasta que otro comience su emisión: este conjunto será para nosotros el turno verbal y cada turno verbal puede incluir uno o varios movimientos.

Los criterios para segmentar movimientos estriban en que se produzca una variación en los componentes analizados en posición de propuesta, esto es, que cambie el destinador, que cambie el destinatario o que cambie la solicitud.

$\mathrm{P}$ - $[\text { de una hermana } \mathrm{de}=\text { mi marido }]^{1}$

$\mathrm{T}-[\text { sí lo tengo aquí }]^{2} /\left[\right.$ ¿cómo se llamaba la $=\mathrm{de}=$ su hermana?] ${ }^{3}$

Poniendo entre corchetes lo que pertenece a un mismo movimiento, en el ejemplo anterior aparecen dos criterios de segmentación: segmentación por cambio de interlocutor ( 1 a 2 ) y de destinatario ( 2 a 3). Mientras que:

T- [yo siempre voy a preguntar a los dos/ que los dos me digan lo que ven è??//] $]^{1}[\text { La idea entonces se le ocurrió } a=\text { Agustín y se la comentó a usted }]^{2}$

Es una ségmentación por cambio de solicitud (1 a 2).

En nuestro esquema interaccional cada movimiento ocupa sucesivamente varias posiciones:

\section{PROPUESTA $\rightarrow$ RESPUESTA $\rightarrow$ REPLICA}

De modo que cualquier movimiento según lo situémos en una u otra posición es descrito en base a categorías distintas. 


\subsection{Categorías de propuesta}

En la medida en que el análisis se centra en el estudio de los procesos de control interpersonal, si analizamos un movimiento en posición de propuesta lo consideramos como una dirección para los movimientos posteriores. Veremos en él las inyunciones que promueve y la forma que adopta y nos dispondremos para hacer un análisis en el que no se clasifique ya lo que los actores hacen o dicen sino las relaciones entre lo que cada uno hace o dice con lo que hacen los demás a lo largo de la interacción.

Todo movimiento en posición de propuesta solicita que se ejecute una acción posterior o que se produzca un resultado que va, desde considerar como auténtica una información y utilizarla como tal (en «mañana va a llover» o en «estuve viendo "Querido detective' y tiene unas canciones guapísimas»), hasta cerrar la puerta y poner la calefacción (en «Haz algo, tengo un frío de mil demonios»). A esta faceta del mensaje la denominamos solicitud.

El análisis tiene en cuenta si la petición, o inyunción, que conforma la solicitud ha sido realizada de forma poco clara para el observador, en el sentido de que el interlocutor se haya interrumpido antes de concretarla mínimamente o porque en el registro no sea distinguible parte o todo el turno verbal, en este caso se puntúa la solicitud como indiscernible.

En el caso en que la solicitud sea discernible se tiene en cuenta su grado de constricción, es decir, si adopta el modo de pregunta u orden (si requiere la realización de acciones concretas) o si por el contrario toma una forma narrativa o explicativa, o si efectúa una descripción o predicción (si solicita acciones difusas en torno a la aceptación de la información transmitida). En el primer caso hablamos de requerimiento y en el segundo de no-requerimiento.

Dentro aún de la categoría solicitud se distingue si ésta es activa o inactiva. Entendemos que una solicitud de un movimiento es activa cuando el destinador marca con señales no verbales (tono, mirada y postura) que su aportación terminó dejando el turno verbal libre para que otro interlocutor efectúe la suya. En cambio la solicitud es inactiva cuando el destiandor no indica que haya concluido su turno y que otro pueda comenzar su intervención (Duncan y Fiske, 1977).

De cada movimiento en posición de propuesta, el S.A.I.I. recoge además de las categorías de solicitud, las de turno verbal y orientación.

El turno verbal no es una unidad lingǘstica sino interaccional que encierra una multiplicidad de registros semióticos que van desde frases a risas, movimientos corporales... Como Sacks, Schegloff y Jefferson (1974) afirman, en una conversación la producción del discurso adopta una organización en turnos verbales y cada turno se construye de forma que se pueda operar la selección del turno siguiente. Hay espacios para marcar el comienzo o el fin de turno como señalábamos en la categoría actividad de solicitud. En el turno verbal se recoge la selección que se ejcuta en cada enunciado del hablante siguiente. Turno verbal (TV) recoge quién es el destinador de la solicitud y a quién se la dirige (destinatario).

En orientación, finalmente, se señala si el destinador mira al destinatario, a la audiencia o a ambos interlocutores o si no mira a ninguno de ellos mientras realiza su solicitud.

En la Tabla II se enumeran los códigos de propuesta para tres interlocutores: $\mathrm{T}, \mathrm{C} 1$ y $\mathrm{C} 2$. «O» significa que el destinador no se orienta hacia ninguno de ellos. 
TABLA II

Códigos de la posición de propuesta

\begin{tabular}{llllll}
\hline \multicolumn{2}{c}{ Turno verbal } & \multicolumn{2}{c}{ Orientación } & \multicolumn{2}{c}{ Solicitud } \\
\hline T a C1 & $(1)$ & No puntuable & $(0)$ & Requerimiento activado & $(1)$ \\
T a C2 & $(2)$ & T mira a C1 & $(1)$ & Requerimiento inactivo & $(2)$ \\
T a C1 y C2 & $(3)$ & T a C2 & $(2)$ & No-requerimiento activado & $(3)$ \\
C1 a C2 & $(4)$ & T a C1 y C2 & $(3)$ & No-requerimiento inactivo & $(4)$ \\
C1 a T & $(5)$ & T a O & $(4)$ & Indiscernible & $(5)$ \\
C1 a C2 y T & $(6)$ & C1 a C2 & $(5)$ & & \\
C2 a T & $(7)$ & C1 a T & $(6)$ & & \\
C2 a C1 & $(8)$ & C1 a T y C2 & $(7)$ & & \\
C2 a T y C1 & $(9)$ & C1 a O & $(8)$ & & \\
& & C2 a T & $(9)$ & & \\
& & C2 a C1 & $(10)$ & & \\
& & C2 a T y C1 & $(11)$ & \\
\end{tabular}

\subsection{Categorías de respuesta}

La siguiente posición en la que puede ser analizado un movimiento es la de respuesta (a una propuesta previa). Al desarrollarse la interacción a través de múltiples canales la respuesta a un movimiento puede adoptar una forma compatible con la emisión de otros movimientos, éste es el caso, por ejemplo, de la respuesta no-verbal de atención que se desarrolla simultáneamente al turno verbal de otro destinator. Pero también ocurre que, para la correcta emisión de un mensaje, puede ser necesario que el canal de transmisión de la información esté libre de otras emisiones, éste es el caso de las emisiones lingüísticas que, salvo excepciones, no se pueden producir simultáneamente por largo tiempo en una conversación. Teniendo esto en cuenta los movimientos en posición de respuesta serán analizados con las categorías de respuesta en turno simultáneo y las categorías de respuesta en turno alternante.

Las categorías de respuesta se refieren a las distintas franjas inyuntivas que pueden observarse en relación con el movimiento anterior tomado como propuesta. Estas inyunciones de la propuesta pueden ser obedecidas o desobedecidas de muy distintas maneras en la respuesta y nos proporcionan la -base de cada categoría.

\subsubsection{Respuesta en turno simultáneo}

Estas categorías recogen exclusivamente conducta no-verbal. Un mensaje emitido en presencia de otros sujetos se realiza inicialmente de modo que resulta justificado decir que pide atención a sus interlocutores al tiempo que los selecciona en su ejecución: se habla a alguien para que, al menos, el otro te escuche. Como Argyle y Cook (1976) decían, el significado fundamental de la mirada es que el otro esté atento, que su canal visual esté abierto. Otros autores como Goodwin (1980)-han estudiado la mirada como criterio de éxito en la comunicación, demostrando el rol esencial que la mirada del oyente juega en la construcción coherente del discurso. 
Atención es la categoría que corresponde a la respuesta en turno simultáneo y responde a una inyunción básica de cualquier movimiento en posición de propuesta que puede ser formulada como «escúchame» (debe ser escuchado). Nuestra categoría atención recoge la orientación del tronco y cabeza del destinatario y la audiencia así como la dirección de la mirada. Atención se puntúa por separado para el destinatario marcado por el movimiento propuesta y para la audiencia. En el caso de que el comportamiento de un sujeto sufra variaciones a lo largo del movimiento es el comportamiento desarrollado durante más tiempo el que se codifica en atención.

Para tres interlocutores atención se codifica según la Tabla III.

TABLA III

Códigos de la posición de respuesta en turno simultáneo

\begin{tabular}{llllll}
\hline $\mathrm{T} a \mathrm{C} 1$ & $(1)$ & $\mathrm{C} 1 \mathrm{a} \mathrm{C} 2$ & $(4)$ & $\mathrm{C} 2 \mathrm{a} \mathrm{T}$ & $(7)$ \\
$\mathrm{T}$ a C2 & $(2)$ & $\mathrm{C} 1 \mathrm{a} \mathrm{T}$ & $(5)$ & $\mathrm{C} 2 \mathrm{a} \mathrm{C} 1$ & $(8)$ \\
$\mathrm{TaO}$ & $(3)$ & $\mathrm{C} 1 \mathrm{a} \mathrm{O}$ & $(6)$ & $\mathrm{C} 2 \mathrm{aO}$ & $(9)$ \\
\hline
\end{tabular}

\subsubsection{Respuesta en turno alternante}

Como ya hemos dicho, a todo movimiento se le dan códigos de propuesta y de respuesta en turno simultáneo pero hay algunos que no reciben códigos de respuesta en turno alternante. Las categorías de RTA se aplican a los "movimientos" siguiendo estas reglas:

a) Si $m_{0}$ es el movimiento analizado y $m_{-1}$ es el movimiento anterior: 1 ) $\mathrm{m}_{0}$ recibirá códigos de RTA siempre que tenga un destinador distinto a $\mathrm{m}_{-1}$; ii) si $\mathrm{m}_{-1}$ tiene dos destinatarios de puntúan los $\mathrm{m}_{0}$ de ambos siempre que sean consecutivos y tengan como destinatario al destinador de $m_{-1}$.

b) Si dos movimientos se realizan simultáneamente $\left(\mathrm{m}_{0} \mathrm{y} \mathrm{m}_{0^{\prime}}\right)$ ambos se relacionarán como respuesta con el movimiento anterior $\left(\mathrm{m}_{-1}\right)$, pero no entre ellos.

c) Cuando un turno verbal tiene dos movimientos con el mismo destinatario se codifican los dos respecto al movimiento anterior $m_{-1}$ y si el segundo movimiento tiene un destinatario distinto éste no se codifica como respuesta a $\mathrm{m}_{-1}$ y queda sin código de $\mathrm{RTA}$.

Las categorías de respuesta en turno alternante son:

A) Direccionalidad y ocupación de canal: El turno verbal como pauta organizativa de una conversación y la actividad de la solicitud delimitan dos líneas inyuntivas cuya respuesta vamos a considerar en las categorías Direccionalidad (DIR) y Ocupación de Canal (OCAN). Ambas categorías tienen como precedente los estudios de Sacks, Schegloff y Jefferson (1978), sobre las técnicas de construcción y atribución de turnos empleados en las conversaciones cotidianas, así como las investigaciones de Duncan y Fiske (1977).

Una inyunción implícita, pero básica, en la toma de palabra es que el destinador pueda completar su intervención hasta que ceda el turno verbal a otro interlocutor. La respuesta a esta inyunción que, formulada negativamente, es «no me interrumpáis» o «dejadme acabar», es una respuesta de tipo cooperativo y la coordinación en los traspasos de los turnos verbales es lo que 
mide ocupación de canal. La OCAN se puntúa como "respetada» cuando el interlocutor que está ocupando el canal verbal completa su movimiento y cede el turno siguiente a otro interlocutor, y se considera "no respetada" cuando antes de que el destinador señale el fin de su turno verbal otro interlocutor inicia su emisión. La OCAN no respetada cubre interrupciones, superposiciones y habla simultánea.

La sucesión de los turnos verbales tiene también otro componente inyuntivo que es la designación del siguiente emisor por el anterior. En TV nosotros recogíamos tanto el destinador como el destinatario elegido por éste; ahora, en direccionalidad tendremos en cuenta el éxito de esta propuesta. Así se puntúa «aceptada» cuando el destinador siguiente coincide con el destinatario del movimiento anterior al que es respuesta; en caso contrario se puntúa direccionalidad "rechazada».

B) Cumplimiento: Las categorías de CUMPLIMIENTO (CUMP) se refieren a la adecuación de movimientos respuesta $\left(\mathrm{m}_{0}\right)$ a las especificaciones de la acción, o resultado, solicitada en el movimiento anterior considerado como propuesta.

Ante una solicitud anterior el movimiento respuesta puede realizar lo que en ella se pide, en este caso hablamos de cumplimiento (que rotularemos CUM y no CUMP para distinguirlo, como subcategoría, de la categoría global): una respuesta es un cumplimiento (CUM) no sólo cuando ejecuta la acción solicitada (respuesta a un requerimiento) sino también cuando utiliza los datos procedentes del mismo (respuesta a un no-requerimiento), ya que al utilizarlos afirman implícitamente su conformidad con ellos.

Otra opción será no ejecutar lo solicitado en ese movimiento pero comprometerse a su cumplimiento posterior. En este caso se aplaza la ejecución de lo solicitado a un momento posterior, ya porque se indica que no se puede cumplir debido a un fallo en la comprensión o en la cualificación del destinatario, ya porque se abre una secuencia paralela que prepara la respuesta posterior. A esta opción la denominamos aplazamiento (APLA).

Cuando en $m_{0}$ se comenta explícitamente que no se va a ejecutar lo solicitado o no se está conforme con la descripción, predicción o explicación dada en $m_{-1}$ se produce un rechazo (RECH) de la solicitud anterior. En el caso en que no se ejecuta lo solicitado, ni se utiliza la información del movimiento a que es respuesta pero no se explicita esto, nos encontraremos, no con un rechazo, sino con un incumplimiento (I).

Por último $m_{0}$ puede resultar ser una respuesta que confirma la recepción y comprensión de la anterior propuesta sin ejecutar lo solicitado. En este caso hablaremos de notificación. En un principio la notificación aparecería como respuesta a la inyunción "presta atención» y por tanto tendría que considerarse como parte de la categoría atención, que en respuesta en turno simultáneo se refiere precisamente a esa franja inyuntiva, pero la notificación (verbal o cuando menos vocálica) como movimiento tiene un aspecto de "solicitud» (se analiza siempre como propuesta) que no tiene la atención. Así un «hum», «uhum» o un «ya», puede proponer al interlocutor que siga con su intervención o que pare. Por otra parte las señales no-verbales en las que se basa atención no informan de la ausencia de problemas de comprensión mientras que la notificación sí lo hace. «Uhum», como respuesta equivalente a «entendido» y como propuesta a «sigue» o "para». 
En los casos en que la solicitud de $\mathrm{m}_{-1}$ consiste en que el destinatario actúe según la información proporcionada (lo que llamaremos no-requerimiento) es fácil confundir NOT y CUM pero la NOT no compromete al destinador con la información, el CUM sí. La NOT no afirma la verdad, o lo acertado o valioso de predicciones, explicaciones, descripciones, etc., el CUM sí.

Veámoslo con ejemplos:

A- (dirigido a B y C) (Bueno quiero que me digáis en vuestra opinión cuál es el problema para el que queréis que os ayudemos] $]^{1}$

$\mathrm{B}$ - (dirigido a C) [¿quién empieza tú o yo?] ${ }^{2}$

C- (a B) [empieza tú]

B- [vale $]^{4}$. [para mí el problema es... $]^{5}$

De 2 a 1 hay un aplazamiento. De 3 a 2, de 4 a 3 y de 5 a 1 hay cumplimiento.

A - (dirigido a B) [para ti Marisa ¿cuál es el problema?] ${ }^{1}$

$\mathrm{B}-$ (dirigido a $\mathrm{A}$ ) [no yo vengo de acompañante ${ }^{2}$

De 2 a 1 RECH (y CU-DIO, como veremos más adelante).

$A$ - (dirigido a B) [¿qué le dijo a usted $C$ ? ¿cómo se lo explicó?] ${ }^{1}$

$\mathrm{B}$ - (dirigido a $\mathrm{A}$ ) [no cuando $\mathrm{D}$ le dijo eso a $\mathrm{C}$ yo no estaba] ${ }^{2}$

$\mathrm{C}$ - (dirigido a B) [no no estabas pero te lo expliqué yom»]

$\mathrm{B}-$ (dirigido a C) [sí bueno a mí me lo explicaste tú ${ }^{4}$

De 2 a 1 I, de 3 a 2 RECH, de 4 a 3 CUM.

C) Inteligibilidad: La inteligibilidad se refiere al comentario implícito o explícito del autor de $\mathbf{m}_{0}$ sobre lo comprensible o incomprensible de $\mathrm{m}_{-1}$.

El señalamiento de fallos en la inteligibilidad de la propuesta puede referirse a un fallo en la recepción o tipificación de la señal (FINT RETEX), o en la comprensión léxico gramatical (FINT COLEG), o por último a un fallo en la comprensión inyuntiva (FINT COIN). Este último fallo en la transmisión de la solicitud puede referirse tanto a la determinación del resultado o la acción solicitada (COIN:R) como a una fallo en la determinación del actor que debe ejcutar la acción solicitada (COIN:DIO).

A- [¿qué opinan de ello?] ${ }^{1}$

$\mathrm{B}$ - [perdón ca quién se lo pregunta?]

$\mathrm{A}-[\mathrm{a} \text { los dos }]^{3}$

\section{De 1 a 2 APLA FINCOIN:DIO.}

$$
\begin{aligned}
& \text { A- [humm a ver/ ¿qué es lo que va mal?] }]^{1} \\
& \text { B- [bueno/ ¿respecto a Antonio?] }]^{2} \\
& \text { A- [uhum }]^{3} \\
& \text { B- [el niño ¿no? }]^{4} \\
& \text { A- [si bueno por lo que queráis consultar }]^{5}
\end{aligned}
$$

De 2 a 1 APLA FINCOIN:R, de 3 a 2 CUM; de 4 a 3 APLA FINCOIN:R.

D) Cualificación: Las categorias de cuestionamiento de la cualificación 
hacen hincapié en la atribución de derechos y deberes, en el saber, querer o poder hacer que en un movimiento se adjudican tanto al destinador, como al destinatario y a la audiencia. En su discurso los actores se cualifican a sí mismos y a los otros como queriendo hacer algo, o sabiendo hacerlo o teniendo el derecho a hacerlo, y sus interlocutores pueden aceptar o no esa cualificación. En todo caso nuestra categoría recoge sólo la aceptación o rechazo de las cualificaciones que se refieren al hacer dentro de la interacción misma, a la producción de intervenciones dentro de la conversación analizada y no las cualificaciones para el hacer o decir extra-conversacional.

Se habla de descualificación para señalar las respuestas en que se ponga en duda la capacidad del actor para producir un resultado o acción, o una solicitud, así como su derecho a hacerlo. De este modo tenemos dos subcategorías descualificatorias: la descualificación del destinatario a la solicitud $\mathrm{m}_{-1}$ y la descualificación del destinador ( $\left.\mathrm{CU}^{-} \mathrm{DIO}, \mathrm{CU}^{-} \mathrm{DOR}\right)$.

A- [hum ¿qué esperáis que consigamos aquí?]]

$\mathrm{B}-[\text { hum (sonríe) yo no lo sél no lo sé }]^{2}$

De 2 a 1 RECH CU-DIO.

$\mathrm{A}-[\text { humm ¿qué essperáis que consigamos aquí? }]^{1}$

$\mathrm{B}-\left[\right.$ no sé por qué pregunta eso, eso lo tendrá que saber usted] ${ }^{2}$

De 2 a 1 RECH CU-DOR.

En el caso en que los interlocutores «eleven» la cualificación propuesta en la solicitud se habla de cualificaciones y, al igual que con las anteriores, en la interacción se puede realizar una cualificación del destinador de la solicitud o una cualificación del destinatario (CU ${ }^{+}$DOR,CU $\left.{ }^{+} \mathrm{DIO}\right)$.

\subsection{Categorías de réplica}

Por último los movimientos reciben un cuarto tipo de categorías que se corresponden con la posiciónde réplica. Con el se analiza el movimiento como evaluación de una propuesta anterior respecto de otra propuesta, inmediatamente precedente o alejada en el tiempo.

Un movimiento $m_{0}$ es categorizado en posición de réplica cuando en él se evalúe el movimiento anterior $\mathrm{m}_{-1}$ como adecuado o inadecuado respecto a una solicitud $\mathrm{m}_{-2}$ anterior a éste un poco más alejada en el tiempo. A esto hace referencia la categoría valoración.

Los criterios para la categoría de valoración son: (i) localizar un movimiento solicitud $m_{-2}$ y (ii) licalizar luego un comentario explícito o implícito que evalúe $m_{-1}$ como respuesta suficiente $o$ insuficiente ante la solicitud $m_{-2}$.

Las categorías de valoración son: valoración negativa implícita, valoración negativa explícita, valoración positiva implícita y valoración positiva explícita.

Los códigos de valoración hacen referencia pues, a su aspecto de crítica o apoyo, a la forma que adopta en el discurso, y también señalan los movimientos de respuesta y propuesta que evalúan. Así los códigos para tres movimientos A, B y C serían: 1) $\mathrm{V}^{-\mathrm{IM}}{ }_{\mathrm{c}(\mathrm{B}-\mathrm{A})}$; 2) $\mathrm{V}^{+\mathrm{IM}}{ }_{\mathrm{c}(\mathrm{B}-\mathrm{A})}$;3) $\mathrm{V}^{-\mathrm{EX}}{ }_{\mathrm{c}(\mathrm{B}-\mathrm{A})}$; 4) $\mathrm{V}^{+\mathrm{EX}} \mathrm{c}_{(\mathrm{B}-\mathrm{A})}$. Que se leen así; 1) $C$ valora implícitamente como insuficiente la respuesta $B$ 
ante la propuesta A. 2) C valora de forma implícita como suficiente la respuesta $B$ ante la propuesta $A$ y así sucesivamente.

Ejemplos de la aplicación de esta categoría son:

$$
\begin{aligned}
& \text { A- [humm a ver/ ¿qué es lo que va mal?] }{ }^{1} \\
& \text { B- [bueno/ ¿respecto a Antonio?] }{ }^{2} \\
& \text { A- [uhum }]^{3} \\
& \text { B- [el niño ¿no? }]^{4} \\
& \text { A- [sí bueno por lo que queráis consultar }]^{5}
\end{aligned}
$$

En 4 respecto a 3 y 2 hay una $\mathrm{V}^{-\mathrm{IM}_{4(3-2)}}$.

$$
\begin{aligned}
& \text { A- (a B) [¿qué le dijo a Vd. C? ¿cómo se lo explicó?] }{ }^{1} \\
& \text { B- (a A) [no cuando D le dijo eso a C ya no estaba] }{ }^{2} \\
& \text { C- (a B) [no no estabas pero cómo te lo expliqué yo yo yo] }{ }^{3} \\
& \text { B- (a C) [sí bueno a mí me lo explicaste tú] }{ }^{4}
\end{aligned}
$$

En 3 respecto 2 y 1 hay una $\mathrm{V}^{-\mathrm{IM}_{3(2-1)}}$.

Implícita y explícita se refieren a la forma que adopta la valoración en el discurso. Si se indican las razones por la que la respuesta se considera insatisfactoria (en el caso de la valoración negativa) la valoración es explícita y si no se indican implícita. Por ejemplo si un interlocutor inicia una intervención de respuesta a otro movimiento y otro interlocutor no toma este movimiento como tal respuesta, este último valora implícitamente de forma negativa la respuesta del orro.

$$
\begin{aligned}
& \text { A- [¿qué os llevó a// pedir ayuda en este momento?] }{ }^{1} \\
& \mathrm{~B} \text { - [hombre que vemos qu e no está que vamos que }]^{2} \\
& \text { C- } \quad \text { [que en vez de ir] }]^{3} \\
& \text { A- }[\text { (hace una señal con la mano alzada a C) }]^{4} \\
& \text { C- } \\
& \text { A- } \\
& \left.\left[\text { es } \int \text { que }\right]^{5} \text { [espere uno de cada veż }\right]^{6}
\end{aligned}
$$

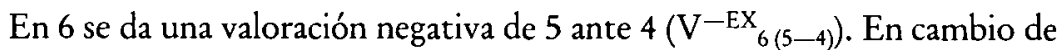
4 a 3 hay un $\mathrm{RECH}$, pero no una valoración negativa de 3 ante $2\left(\mathrm{~V}^{+\mathrm{IM}} \mathrm{M}_{4(3-2)}\right)$.

En la Tabla IV se enumeran las categorías de respuesta en turno alternante y réplica.

TABLA IV

Códigos de la posición de Respuesta en turno alternante y réplica

\begin{tabular}{llllll}
\hline $\begin{array}{c}\text { Direcciona- } \\
\text { lidad }\end{array}$ & $\begin{array}{c}\text { Ocupación } \\
\text { de canal }\end{array}$ & \multicolumn{1}{c}{$\begin{array}{c}\text { Cumpli- } \\
\text { miento }\end{array}$} & \multicolumn{1}{c}{$\begin{array}{c}\text { Inteligi- } \\
\text { bilidad }\end{array}$} & Cualificación Valoración \\
\hline 1. DIRACEP & 1. OCANACEP & 1. CUM & 1. NFIN & 1. NCU & 1. V IM \\
2. DIRNEG & 2. OCANEG & 2. I & 2. RETEX & 2. CU DIO & 2. V IM \\
& & 3. NOT & 3. COLEG & 3. CU-DOR & 3. V-EX \\
& & 4. RECH & 4. COIN:R & 4. CU DIO & 4. V EX \\
& & 5. APLA & 5. COIN:DIO & 5. CU DOR & 5. NOCAT \\
& & 6. NOCAT & 3OCAT & 6. NOCAT & \\
\hline
\end{tabular}




\section{FIABILIDAD INTERJUECES}

La fiabilidad del S.A.I.I. fue comprobada con motivo de la investigación sobre entrevistas terapéuticas que se expone en el siguiente apartado. Los estadísticos utilizados para calcular la fiabilidad interjueces de las distintas categorías fueron dos: un índice de porcentaje de acuerdo en puntajes y el índice Kappa desarrollado por Cohen (utilizado únicamente para las categorías de réplica y de RTA). Para las categorías de respuesta en turno simultáneo (RTS) los índices se calcularon sobre las codificaciones independientemente de dos jueces. Para el resto de las categorías los jueces fueron tres.

El índice modificado de acuerdos-desacuerdos, referido siempre a la categorización de un mismo movimiento por los distintos jueces, tuvo los siguientes resultados:

1. Segmentación de movimientos y turnos verbales: Indice acuerdo/desacuerdo $97,88 \%$.

2. Turno verbal: Indice acuerdo/desacuerdo $99,71 \%$.

3. Solicitud: a) Discernibilidad de la solicitud: Indice $a / d=99,42 \%$. b) Constricción: Indice $a / d=97,4 \%$. c) Actividad: Indice $a / d=$ 97,21\%.

4. Atención: Indice de acuerdo/desacuerdo $=95,5 \%$.

5. Segmentación de pares para las categorías de $R T A$ : Indice $\mathrm{a} / \mathrm{d}=$ $97,91 \%$ (sin contar las diferencias producidas por 1 ). Indice $\mathrm{a} / \mathrm{d}=$ $94,22 \%$ (contando también las diferencias producidas por 1 ).

6. Direccionalidad: Las producidas por 1 y 2.

7. Ocupación de canal: No hubo diferencias.

Como las categorías restantes necesitaban introducir correcciones que no sólo tuviesen en cuenta el efecto del azar sino también su probabilidad de aparición y además se trataba de categorías nominales nos decidimos a utilizar el estadístico Kappa que Cohen (1960) desarrolló para escalas norminales y que siguiendo a Anguera (1983) llamaremos índice de porcentaje de acuerdo corregido. Las puntuaciones Kappa obtenidas para cada categoría fueron halladas tanto para tablas que incluían los indiscernibles como para tablas que los excluían.

Los resultados para los 946 pares segmentados (sin indiscernibles) fueron:

$\begin{array}{lll}\text { Cumplimiento: } & P_{0}=0,963 & \\ & P_{\mathrm{c}}=0,4735 & \text { Kappa }=0,929 \\ \text { Inteligibilidad: } & P_{0}=0,997 & \\ & P_{\mathrm{c}}=0,926 & \text { Kappa }=0,981 \\ \text { Cualificación: } & P_{0}=0,9936 & \\ & P_{\mathrm{c}}=0,9768 & \text { Kappa }=0,724 \\ \text { Valoración: } & P_{0}=0,9767 & \\ & P_{\mathrm{c}}=0,8671 & \text { Kappa }=0,825\end{array}$

Aunque no se han establecido criterios muy claros en torno a la puntuación apropiada de las pruebas de fiabilidad interjueces ni hay acuerdo en hacerlo (Pinsoff, 1981), Hartmann (1982) sugiere que los valores recomendados de 
fiabilidad oscilan entre 0,60 y 0,75 para la Kappa de Cohen y entre 0,70 y 0,90 para el índice acuerdo-desacuerdo.

Las categorías del S.A.I.I. cumplen sobradamente estos criterios siendo la categoría más difícil la cualificación. Su dificultad vino de la tendencia a codificar descualificaciones del destinatario donde otros jueces no encontraban cuestionamiento de cualificación.

En el proceso de categorización hubo un tipo de respuesta que produjo problemas de acuerdo entre los jueces, se trataba de respuestas a un interlocutor que implicaban una pregunta al otro interlocutor. Veámoslo:

T- [ia últimos de octubre te lo dijo?]'

A- [no recuerdo eh] $]^{2}$

T- $[\text { hum (risa) }]^{3}$

A- [yo sé que hace ya cierto tiempo// bueno/ pues desde que viniste la última vez que fue/ ya paso $=$ tiempo dos semanas ¿no? $]^{4}$

B- $\quad$ [que vendría el dos o el tres aquí ino? a tomar/ que me tomaran datos y eso $]^{5}$

$\mathrm{T}-\left[\mathrm{si}^{\prime} / \text { debió ser por ahí }\right]^{6}$

Los pares 3-4 y 4-5 reproducen este patrón de respuesta que se analizó de la siguiente forma:

$\mathrm{T}$ pide que A realice un comportamiento X; A responde con un mensaje que constituye una solicitud idéntica a $B$ por lo que, al abrir una secuencia lateral, se trataría de un aplazamiento, y, al dirigir la solicitud a otro interlocutor, se descualificaría a sí mismo como destinatario. Esta respuesta la repite B respecto al movimiento 4 .

La codificación quedaría entonces así:

2-1 DIRACEP, OCANACEP, RECH, NOFINT, CU--DIO, $\mathrm{V}^{+1 \mathrm{M}}$

3-2 DIRACEP, OCANACEP, CUM, NOFINT, NOCU, $\mathrm{V}^{+\mathrm{IM}}$

4-3 DIRACEP, OCANACEP, APLA, NOFINT, CU-DIO, $\mathrm{V}^{+\mathrm{IM}}$

5.4 DIRACEP, OCANEG, APLA, NOFINT, CU-DIO, $\mathrm{V}^{+\mathrm{IM}}$

\section{CONCLUSION}

En El caballo mágico, Idries Shah (1977) recoge una alegoría árabe del siglo XIV, titulada «Las hormigas y la pluma», en la que relata los maravillosos hallazgos de unas hormigas que, concienzuda y minuciosamente, descubren poco a poco el origen de los hermosos garabatos que trazaba una pluma sobre la superficie de un papel. Una tras otra van descubriendo el misterio: una los dedos que rodean e impulsan la pluma, otra la mano de la que forman parte, otras muchas el brazo y, tras el brazo, el cuerpo entero (con sus dos brazos y sus dos piernas). Con tenacidad y deleite llegan a conseguir una idea adecuada de la mecánica de la escritura:

Pero - dice el narrador - acerca del sentido e intención de la escritura, y de cómo, en último término, eran éstos determinados, no pudieron saberlo a través de su acostumbrado método de investigación. Porque no sabían leer y escribir. 
Cualquiera que use el S.A.I.I. hará bien en acordarse de las hormigas. Una y otra vez, cuando elaborábamos los siete sistemas de codificación que fuimos probando y descartando hasta concretar los análogos y luego el sistema de códigos, nos chocaba la enorme riqueza de la interacción cara a cara y la pobreza de todos nuestros intentos de reducirla. Esa impresión no desapareció en absoluto cuando, ya probada la fiabilidad y exhaustividad del sistema de categorías, emprendimos la codificación de entrevistas. Sin embargo, cuando por fin llegó la hora de analizar los datos resultantes de la categorización, la impresión cambió radicalmente en sentido contrario: un análisis pobre desde el punto de vista de ruestras competencias como actores, resulta exasperantemente complejo desde el punto de vista de un usuario de programas informáticos de tratamiento de datos. Una vez terminada esta etapa, y obtenidos los resultados de la investigación, el sistema de análisis nos volvió a parecer exiguo.

En cierta medida una descripción obtenida con el S.A.I.I. es en el Análisis de la Interacción lo que en Economía pudiera ser un informe en el que aparezcan el número de transacciones intentadas y el número de transacciones finalmente realizadas en una región, pero que no especifique ni el tipo o la naturaleza de las mercancías intercambiadas ni el volumen económico que ha supuesto, cada transacción por separado y todas las transacciones en conjunto. Pretender dar cuenta de la marcha de la economía con una descripción de ese estilo sería ridículo. Pero querer hacerlo sin ser capaces de distinguiir en qué momentos se ofrecen las mercancías y en que momentos se realizan las transacciones sería estúpido. Cuando las hormigas descubren por fin que las marcas son producidas por un ser humano están muy lejos de saber leer, pero ya han tomado la senda que lleva a la lectura. Sin la larga y minuciosa investigación previa la lectura sería, para ellas, mucho más inconcebible.

\section{Referencias}

Anguera, M. T. (1983). Manual de prácticas de observación. México: Trillas.

Argyle, M. y Cook, L. M. (1976). Gaze and Mutual Gaze. Cambridge, Mass.: Cambridge University Press.

Austin, J. L. (1982). How Do Things with Words. Oxford: Oxford University Press. (Orig. 1962).

Bakeman, R. y GotTman, J. M. (1989). Observación de la interacción. Introducción al análisis secuencial. Madrid: Morata. (Orig. 1986).

Bruner, J. (1986). El babla del niño. Aprendiendo a usar el lenguaje. Barcelona: Paidós. (Orig. 1983).

Cohen. J. (1960). A coefficient of agreement for nominal scales. Education and Psychological Measurement, 20: 37-46.

DunCAN, S. Y y Friske, D. W. (1977). Face-Face interaction: Research, methods and theory. Hillsdale, New Jersey: Lawrence Erlbaum Associates Publishers.

EDW ARDS. D. y MERCER, N. (1988). El conocimiento compartido. El desarrollo de la comprensión en el aula. Barcelona: Paidós. (Orig. 1987).

ERviN-TRIPP. S. (1977). Some features of early child-adult dialogues. Language and Society, 7 , 357-373. 
Gúmlz FonTANIL. Y. (1989). Análisis de la interacción: una nueva propuesta y su aplicación al estudio de las entrevistas terapéuticas. Oviedo: Tesis doctoral inédita.

Gomkz Fontanil. Y. y Coto Ezama. E. (1990). Modelos para el análisis de la interacción: un esquema básico para la construcción de categorías. Psicothema, 2 (2).

GoODWIN. C. (1980). Restarts, pauses and the achievement of a state of mutual gaze at turnbeginning. Sociological Inquiry, 50: 3-4.

GotTMan. J. M. (1979). Marital Interaction. Experimental investigations. Nueva York: Academic Press.

HALEY. J. (1977). Descripción interaccional de la esquizofrenia. En: Jackson, D. D. (comp.) Comunicación, familia y matrimonio (pp. 183-206). Buenos Aires: Nueva Visión. (Orig. 1959).

Harre. R. (1982). El Ser Social. Una teoría para la psicología social. Madrid: Alianza Editorial. (Orig. 1979).

HARRE, R. y SECORD, P. F. (1972). The Explatanion of Social Behaviour. Oxford: Blackwell.

Hartmann, D. P. (1982). Using Observers to Study Behavior: New Directions for Methodology of Social and Behavioral Science. San Francisco: Jossey-Bass.

KAYE. K. y FOGEL, A. (1980). The temporal structure of face to face communication between mothers and infants. Developmental Psychology, 16, 454-464.

Lozano. J.; Peña-marín, C. y Abril. G. (1982). Análisis del discurso. Hacia una semiótica de la interacción textual, Cátedra, Madrid.

Metz. A. S. (1965). A Comparison of the Use of the Sound Recording and the Written Transcript in the Coding of Verbal Interaction. Journal of Social Psychology, 65: 325-335.

Miklowitz. D. J.; Goldstein. M. J.; Doane. J. A.; Nuechterlein. K. H.; Strachan. A. M.; Snyder, K. S. y Magaña-Amato, A. (1989). Is Expressed Emotion and Index of a Transactional Procesess? I. Parent's Affective Style. Family Process 28 (2), 153-167.

Mishler. E. G. y WaxLer. N. (1968). Interaction in Families: An Experimental Study of Family Processes and Schizophrenia. Nueva York: John Wiley and Sons.

Penman, R. (1980). Communication Processes and Relationships. Londres: Academic Press.

Pinsoff, W. M. (19811). Family Therapy Process Research. En Gurman, A. S. y Knishern, D. P. (Comp.), Handbook of Family Therapy. Nueva York: Brunner/Mazel.

RISKIN, J. y FAUNCE, E. (1970). Family Interaction Scales. Archives of General Psychiatry 22, 504-537.

Rogers, L. E.; Courthright. J. A. y Millar, F. E. (1979). Relational Control and Dyadic Understanding an Exploratory Predictive Regression Model. En: Nimmo, D. (Comp.), Communication Yearbook 3. New Brunswick, N. J.: TICA.

Sacks, H.; SChegloff, E. y Jefferson, G. (1978). A Simplest Systematics for the Organization of Turn-Taking in Conversation. En Shenkein, J. (Comp.), Studies in the Organization of Conversational Interactional. Nueva York: Academic Press.

SBISA, M. (1984). On Illocutionary Types. Journal of Pragmatics, 8: 93-112.

SнAн, I. (1977). El caballo mágico. Caravana de sueños. México: Fotoedisa. (Orig. 1968).

Simon, A. y Boyer, E. G. (1970). Mirrors for Behavior. Vol. II. Filadelfia: Research for Better Schools Inc.

Sinclair. J. McH. y Coulthard. R. M. (1975). Towards an Analysis of Discourse: The English used by Teachers and Pupils. Londres: Oxford University Press.

Sluzki, C. E.; Beavin, J.; TARnopolsky, A. y Veron, E. (1966). Transacciones descalificadoras. Investigación sobre el «doble vínculo". Acta Psiquiátrica y Psicológica de América Latina 12 (4): 329-343. Recopilado en Watzlawick, P. y Weakland, J. H. (Comp.), The Interactional View (pp. 208-277). Nueva York: Norton and Company, 1977.

Sluzki, C. E. y BeAvin, J. (1965). Simetría y complementariedad: Una definición operacional y una tipología de parejas. Acta Psiquiátrica y Psicológica de América Latina 11 (3), 321-330.

Stubs. M. (1987). Análisis del Discurso. Análisis sociolingüístico del lenguaje natural. Madrid: Alianza Editorial. (Orig. 1983).

Valdes Villanueva, L. M. (Comp.) (1983). Significado y acción. Valencia: Rubio.

Viaro, M. y LeONard, P. (1982). "Le insubordinazioni», Terapia Familiare 12: 41-63.

W Axler, M. H. y Mishler, E. G. (1966). Scoring and reliabilicy problems in interaction process analysis: a methodological note. Sociometry, 29: 28-40. 